\title{
The Role of Vagus Nerve on Dexmedetomidine Induced Survival and Lung Protection in a Sepsis Model in Rats.
}

\section{Yumo Li}

Yuying Children's Hospital of Wenzhou Medical College: Wenzhou Medical University Second Affiliated Hospital

\section{Binbin Wu}

Yuying Children's Hospital of Wenzhou Medical College: Wenzhou Medical University Second Affiliated Hospital

\section{Cong Hu}

Yuying Children's Hospital of Wenzhou Medical College: Wenzhou Medical University Second Affiliated Hospital

Jie Hu

Luoyang Central Hospital Affiliated to zhengzhou university

\section{Qingquan Lian}

Yuying Children's Hospital of Wenzhou Medical College: Wenzhou Medical University Second Affiliated Hospital

Jun Li

Yuying Children's Hospital of Wenzhou Medical College: Wenzhou Medical University Second Affiliated Hospital

\section{Daqing Ma ( $\nabla$ d.ma@imperial.ac.uk)}

Imperial College London https://orcid.org/0000-0003-1235-0537

\section{Research Article}

Keywords: Dexmedetomidine, Vagus nerve, Acute lung injury, Inflammation, Cytokines

Posted Date: May 17th, 2021

DOI: https://doi.org/10.21203/rs.3.rs-483367/v1

License: (c) (i) This work is licensed under a Creative Commons Attribution 4.0 International License.

Read Full License 
1 The role of vagus nerve on Dexmedetomidine induced survival and lung protection in a

2 sepsis model in rats

3 Yumo $\mathrm{Li}^{1}$, Binbin $\mathrm{Wu}^{1^{*}}$, Cong $\mathrm{Hu}^{1,2}$, Jie $\mathrm{Hu}^{3}$, Qingquan $\mathrm{Lian}^{1}$, Jun $\mathrm{Li}^{1}$, Daqing $\mathrm{Ma}^{2^{*}}$

$4 \quad{ }^{1}$ Department of Anesthesiology, Perioperative and Pain Medicine, The Second Affiliated

5 Hospital and Yuying Children's Hospital of Wenzhou Medical University, Wenzhou, Zhejiang,

6 China

$7 \quad{ }^{2}$ Division of Anaesthetics, Pain Medicine and Intensive Care, Department of Surgery and

8 Cancer, Faculty of Medicine, Imperial College London, Chelsea and Westminster Hospital,

$9 \quad$ London, United Kingdom

$10{ }^{3}$ Department of Anesthesiology, Luoyang Central Hospital Affiliated to Zhengzhou University,

11 Luoyang, Henan, China

12

13 *Correspondence:

14 Daqing Ma

15 d.ma@imperial.ac.uk

16 Or

17 Binbin $\mathrm{Wu}$

18 wbb19880117@163.com 
Abstract

Background: Sepsis often results in acute lung injury (ALI). Sedative dexmedetomidine (Dex) was reported to protect cells and organs due to its direct cellular effects. This study aims to investigate the role of vagus nerves on Dex induced lung protection in a model of lipopolysaccharide (LPS)-induced ALI in rats.

Methods: The bilateral cervical vagus nerve of male Sprague-Dawley rats was sectioned or just exposed without section as sham surgery. The ALI was induced by intraperitoneal injection of LPS (1 or $10 \mathrm{mg} / \mathrm{kg}$ ). After LPS administration, Dex antagonist yohimbine (YOH) $(1 \mathrm{mg} / \mathrm{kg})$ and/or Dex $(25 \mu \mathrm{g} / \mathrm{kg})$ was injected intraperitoneally at $0,4,8$ and 12 hours to rats with or without vagotomy. The severity of ALI was determined with survival curve analysis and lung pathological scores of haematoxylin and eosin (H-E) staining sections. The plasma

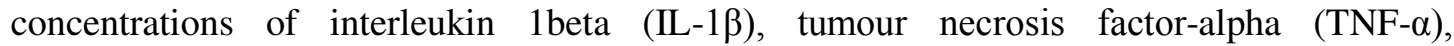
catecholamine (CA) and acetylcholine (Ach) were measured with enzyme-linked immunosorbent assay (ELISA).

Results: The median survival time of LPS-induced ALI rats was significantly prolonged by Dex (22 hours, $50 \%$ CI, $[31.25,90.63])$ compared that in the LPS group (14 hours, $50 \%$ CI, $[18.75,81.25], \mathrm{P}<0.05)$, and the acute lung injury score was significantly reduced by Dex $(6.5,50 \% \mathrm{CI},[5.75,7.5]$ vs $11.5,50 \% \mathrm{CI},[10.75,12.50]$ in the LPS group, $\mathrm{P}<0.01)$. However, these protective effects of Dex were significantly reduced by either $\mathrm{YOH}$ administration or vagotomy. Dex significantly decreased LPS-induced plasma IL-1 $\beta$ (pg/ml) $(20.75 \pm 0.78 v s .30 .22 \pm 2.62, \mathrm{P}<0.01), \mathrm{TNF}-\alpha(\mathrm{pg} / \mathrm{ml})(205.30 \pm 9.39 v s .273 .40 \pm 14.50, \mathrm{P}$ $<0.01)$, and CA $(\mathrm{pg} / \mathrm{ml})(825.70 \pm 43.46$ vs. $1188.00 \pm 64.40, \mathrm{P}<0.01)$ but increased the secretion of Ach $(\mathrm{pg} / \mathrm{ml})(507.20 \pm 49.52$ vs. $296.50 \pm 62.44, \mathrm{P}<0.01)$; these effects of Dex was partially abolished by vagotomy.

Conclusions: Our data suggested that Dex increased vagal nerve tone which partially contributed to its anti-inflammatory and lung protective effects. The indirect anti-inflammation and direct cytoprotection of Dex are likely through high vagal nerve tone and $\alpha_{2}$-adrenoceptor activation, respectively. 
Keywords: Dexmedetomidine, Vagus nerve, Acute lung injury, Inflammation, Cytokines. 


\section{Background}

The autonomic nervous system plays an essential role in inflammation and immune modulation (Benarroch 2019); for example, vagal nerve stimulation can suppress both local and systemic inflammation (Borovikova, Ivanova, Nardi, et al. 2000). Besides, previous studies (Vida et al. 2011; Pavlov and Tracey 2015) also demonstrated that vagus nerve decreased the levels of inflammatory cytokine such as tumor necrosis factor (TNF) and interleukin (IL), which are the key mediator of the inflammatory response in LPS-induced endotoxemia; these effects may be closely associated with its anti-inflammatory effect through the cholinergic anti-inflammatory neural pathway. Indeed, stimulation applied to the vagus nerves elevated the level of acetylcholine, a neurotransmitter, that interacts with $\alpha 7$ subunit of the nicotinic acetylcholine receptor $(\alpha 7 \mathrm{nAChR})$. It was also reported that vagus nerve stimulation or pre-treatment with an $\alpha 7 \mathrm{nAChR}$ agonist reduced neuroinflammation while vagotomy or using selective a7nAChR antagonists enhanced the inflammatory response (Zhu et al. 2016).

Dexmedetomidine (Dex) is a highly selective $\alpha_{2}$-adrenoceptor agonist that has analgesic and sedative effects (Chi et al. 2015; Meng et al. 2018). Dex was demonstrated to inhibit systemic inflammation and oxidative stress, and protect against inflammation and oxidative stress-related diseases (Wang et al. 2017). Clinically, Dex was reported to significantly reduce inflammatory factors IL- $1 \beta$, IL- 6 and TNF- $\alpha$, and intra-abdominal pressure in patients with sepsis after abdominal surgery, compared to propofol (Tasdogan et al. 2009). In addition, previous studies have found that the parasympathetic nerve played an important role in the anti-inflammatory effect of Dex, which may activate the efferent vagus nerve and led to the release of anti-inflammatory factors (Xiang et al. 2014). We, therefore, designed the current study to investigate the role of vagus nerve on Dex against mortality and LPS-induced ALI in rats.

\section{Methods}

Animal acute lung injury model 
Male Sprague-Dawley rats (200-250g, 7 weeks old) were purchased from Shanghai Laboratory Animal Centre (Shanghai, China). All animal experimental procedures were approved by the Institutional Animal Care and Use Committee of Wenzhou Medical University and were performed following the Guide for the Care and Use of Laboratory Animals.

\section{Sepsis model, vagotomy and drug administration}

Rats were anesthetized with intraperitoneal injection of sodium pentobarbital (Zizheng, Shanghai, China) (30 mg/kg) and then randomly subjected to vagus nerves exposure only (sham surgery) in the carotid sheaths through a median skin incision or then cut in both side (vagotomy group). Thirty minutes after surgery, rats were injected intraperitoneally with 1 or $10 \mathrm{mg} / \mathrm{kg}$ lipopolysaccharide (LPS) (Sigma-Aldrich, St. Louis, MO, USA) or an equal volume of normal saline. Rats were injected with Dex (TCI, Shanghai, China) (25 $\mu \mathrm{g} / \mathrm{kg}$ ) intraperitoneally every four hours with a total of 4 injections after LPS administration. Dex antagonist yohimbine (YOH) (Solarbio, Beijing, China) $(1 \mathrm{mg} / \mathrm{kg})$ was injected and followed by Dex injection in some cohorts.

\section{Experimental groups}

In the sham surgery cohorts, they were randomly subdivided into 5 groups according to injections of normal saline, Dex, LPS, LPS plus Dex, or LPS plus YOH and Dex. In the vagotomy cohorts, they were randomly subdivided into 4 groups according to injections of normal saline, Dex, LPS, LPS plus Dex, or LPS plus YOH and Dex. All 9 groups $(n=8)$ were subjected to survival assessment (Fig. 1). Their respiration and locomotor activities were closely monitored.

\section{Blood and lung tissue sampling and analyses}

New cohorts of 9 groups $(\mathrm{n}=6-10)$ illustrated in Fig. 1 were repeated under the identical experimental conditions. After 16 hours experiments, they were sacrificed under terminated anaesthesia of intraperitoneal injection with sodium pentobarbital $(60 \mathrm{mg} / \mathrm{kg})$ anesthesia (Zi et 
al. 2019). Their blood samples via cardiac puncture and the upper lobe of the left lung were harvested for further assessments. The blood samples were clotted for 1 hour and then centrifuged at $3000 \mathrm{rpm}$ for 20 minutes. The serums were subsequently collected to determine the Acetylcholine (Ach), catecholamine (CA), IL-1 $\beta$ and TNF- $\alpha$ with ELISA kits (Yingxin, Shanghai, China) according to the manufacturer's instructions.

Lung tissues were fixed with $10 \%$ paraformaldehyde (Solarbio, Beijing, China). The sample was then embedded in paraffin, sliced into 4- $\mu \mathrm{m}$ sections, and stained with H-E (Solarbio, Beijing, China) and 3 sections/animal were randomly chosen to assess lung morphology (10 fields/section at x20 magnifications) under an Olympus microscope (Olympus BX51/Olympus DP71) in a blinded manner. Severity of lung injury was evaluated with a semi-quantitative scale system from 0 to 3 grades: Grade 0 , normal pulmonary histology; Grade 1, mild moderate interstitial congestion and neutrophil leukocyte infiltrations; Grade 2, moderate neutrophil leukocyte infiltration, perivascular edema formation, and partial destruction of pulmonary architecture; Grade 3, complete destruction of the pulmonary architecture with dense neutrophil leukocyte infiltrations and abscess formation (Chen et al. 2016).

\section{Statistical analysis}

The survivals and ALI scores were expressed as median and 50\% confidence interval (median, $50 \%$ CI $\left[25^{\text {th }}\right.$ percentiles, $75^{\text {th }}$ percentiles $]$ ), which was presented as survival curve and box plot, respectively. The data of ELISA was expressed as mean \pm SD together with dots plot. Statistical analysis was performed with GraphPad Prism 7.0 software (GraphPad Software, La Jolla, California, USA). Survival data were analyzed using a Kaplan-Meier curve and a log-rank test. If normally distributed, the data was analyzed with one-way analysis of variance (ANOVA) followed by Tukey's multiple comparisons test, or otherwise, the Dunnet T3 test was used. A p-value $<0.05$ was considered as a statistical significance. 
The mortality rate of the vagotomy (VAG) group was $100 \%$ at the 80 hours of the experimental period and the median survival time was 14 hours (50\% CI, [12.50, 75.00]); Dex significantly increased survival to $25 \%$ and the median survival time was 24 hours (50\% CI, [12.50, 90.63]) ( $\mathrm{p}=0.008)($ Fig. 2A). To determine the dose effects of LPS on survival, 1 $\mathrm{mg} / \mathrm{kg}$ and $10 \mathrm{mg} / \mathrm{kg}$ LPS were used in rats without vagotomy. The mortality induced by the low dose of LPS with or without Dex and YOH was the same to be $16.7 \%$ and there was no difference of survival between two groups (Fig 2B). However, Dex decreased the mortality to $83.3 \%$ from $100 \%$ induced by the high dose of LPS and increased the median survival time to 22 hours (50\% CI: [31.25, 90.63]) from 14 hours (50\% CI: [18.75, 81.25]) of the high dose of LPS (Fig. 2C). Yohimbine abolished the effects of Dex (Fig. 2C). Dex increased the median survival time to 24 hours (50\% CI: $[6.25,87.50])$ from 15 hours (50\% CI: $[25.00,93.75])$ induced by the low dose of LPS after vagotomy (Fig. 2D). Dex increased the median survival time to 14 hours $(50 \%$ CI: $[25.00,93.75])$ from 5 hours $(50 \%$ CI: $[6.25,75.00])$ induced by the high dose of LPS after vagotomy (Fig. 2E). Yohimbine abolished these effects of Dex (Fig. 2D, E).

\section{The vagus nerve is involving in Dex inhibiting lung pathological injury}

The lung morphology under light microscopy (Fig. 3A-I) showed that the alveolar wall of the lung tissue was thin, and the alveolar structure was clear without congested or widened, and bleeding or inflammatory cell infiltration in the control group and the Dex alone group. There were no severe pathological changes in the VAG group and LPS (10) + Dex group. However, lung interstitium with inflammatory cell infiltration and alveolar wall thickening and congestion were readily noticed after the high dose of LPS injection. Moreover, the lung tissue of the remaining 4 groups showed different degrees of pathological abnormalities, including pulmonary interstitial congestion, edema and hemorrhage, alveolar collapse, and the infiltration of inflammatory cells in the alveolar cavity. The lung injury scores were significantly increased by the high dose of LPS and vagotomy alone in combination which 
was decreased by Dex. Compared with the LPS (10) + Dex group, the lung injury of the LPS (10) $+\mathrm{YOH}+$ Dex group was increased $(12.00,50 \% \mathrm{CI},[10.00,14.25]$ vs. 6.50, 50\% CI, $[5.75,7.50], P<0.01)$. Compared with the VAG + LPS $(10)+$ Dex group, the lung injury of the LPS (10) + Dex group was reduced $(6.50,50 \% \mathrm{CI},[5.75,7.50]$ vs. 10.00, 50\% CI, [8.75, 12.50], $\mathrm{P}<0.01$ ), but there was no significant difference between the VAG + LPS (10) group and the VAG + LPS (10) + Dex group $(10.00,50 \%$ CI, [8.75, 11.25] vs. 10.00, 50\% CI, [8.75, 12.50], $\mathrm{P}>0.05)($ Fig. 3J).

\section{The anti-inflammatory effects of Dex with vagus nerve integrity.}

The high dose of LPS significantly increased both IL-1 $($ Fig. 4A) and TNF- $\alpha$ (Fig. 4B) compared with the control group (IL-1 $1 \beta, 30.22 \pm 2.62$ vs. $18.01 \pm 2.28$, p < 0.01; TNF- $\alpha$, $273.40 \pm 14.50$ vs. $180.00 \pm 30.96, \mathrm{p}<0.01)$. Dex significantly decreased the increases of both cytokines induced by LPS. Yohimbine revised the effect of Dex. Vagotomy alone or superposed with LPS and/or Dex had no further effects on these measurements (Fig. 4).

\section{The effects of Dex and VAG on neurotransmitters}

Vagotomy and LPS significantly decreased acetylcholine (Ach) from $578.00 \pm 59.05$ in the controls to $368.60 \pm 54.40$ and $296.50 \pm 62.44$ ( $<<0.01)$, respectively. This reduction was significantly reversed by Dex regardless of YOH (LPS + Dex, 507.20 \pm 49.52 ; LPS + YOH + Dex, $462.20 \pm 51.69$,). Vagotomy maintained Ach at a low level after superposed with LPS and Dex alone and in combination (Fig. 5A). Dex significantly decreased $(506.40 \pm 105.00)$ but LPS significantly increased catecholamine (CA) (1188.00 \pm 64.40$)$ when compared that in the controls $(713.00 \pm 133.90)(\mathrm{p}<0.01)$. Dex decreased an increase of CA induced by LPS. Compared to vagotomy alone (581.80 \pm 61.37$)$, vagotomy maintained CA at a high level after superposed with LPS and Dex alone and in combination (VAG + LPS, $945.90 \pm 93.45$; VAG + LPS + Dex, $1022.00 \pm 98.24)($ Fig. 5B).

\section{Discussion}

In the current study, the LPS-induced lung injury model was established in rats with injection 
of 1 or $10 \mathrm{mg} / \mathrm{kg}$ concentration LPS. It was found that Dex significantly decreased LPS-induced cytokine release, protected acute lung injury and prolonged survival. These effects of Dex against LPS were attenuated after vagotomy, indicating that the protective effects of Dex partially through vagus nerve tonic anti-inflammatory effects. Dex inhibited the increase of CA and revised the decrease of Ach that was induced by LPS and these effects of Dex were abolished by vagotomy. This implies that the vagus nerve offers an organ-protective effect, and the protective effects of Dex is, at least in part, related to the integrity of the vagus nerve.

LPS activates macrophages and other inflammatory cells, resulting in uncontrolled release of inflammatory cytokines, which in turn causes pulmonary oedema, refractory hypoxemia and pulmonary hypertension, and further develops into ALI (Su et al. 2004). It has been reported that Dex alleviated ALI through suppressing the immune response (Zhang et al. 2017). The possible mechanism of the anti-inflammatory effect of Dex is to activate the medulla oblongata $\alpha_{2}$-adrenergic receptor signaling pathway, causing the peripheral vagus nerve to release Ach and promote the release of Ach and acting on $\alpha 7 \mathrm{nAChR}$. In one hand, the activation of $\alpha 7 \mathrm{nAChR}$ activates Jak kinase 2 to inhibit the production of reactive oxygen species and cytokines; on the other hand, it activates the PI3K/Akt signaling pathway to produce a strong anti-inflammatory effect (Egea et al. 2015; Lankadeva et al. 2019). These suggested that the protective effects afforded by Dex at least in part through the integrity of vagus nerve. It is very true that vagal nerve tonic inhibition of high sympathetic drive is an important mechanism to maintain body hemostasis for keeping lives (Bonaz, Sinniger, and Pellissier 2017), whilst its anti-inflammation, and immuno- and organo-protections have been well reported in the literature (Borovikova, Ivanova, Nardi, et al. 2000) . Therefore, it was not surprisingly found in the current study that vagotomy resulted in a very high mortality of rats, more severe LPS-induced lung injury and an inhibition on Dex protective effects to LPS-induced ALI. In addition, the vagus nerves regulate the body's immune response to inflammation (Kaniusas et al. 2020; Pavlov and Tracey 2012). It has been well documented that vagus nerve stimulation can suppress both local and systemic inflammation (Borovikova, 
Ivanova, Zhang, et al. 2000). Indeed, Dex was well reported to increase parasympathetic nerve tone and provided its protection against kidney injury (Ma et al. 2020).

Our results showed that Dex significantly reduced the increase of cytokines (IL-1 $\beta$ and TNF- $\alpha$ ) that were induced by LPS while vagotomy inhibited these effects of Dex. IL- $1 \beta$ and TNF- $\alpha$ can cause a series of pathological changes, including inflammation, cell senescence, autophagy and apoptosis (Wang et al. 2020). LPS produces self-amplification loops after monocyte/macrophage activation and activates inflammasomes to release mature IL-1 $\beta$ through NLRP3/caspase-1 cascade (Cavaillon 2018; Li et al. 2018). Accumulating evidence indicated that the release of pro-inflammatory cytokines played an important role in ALI (Zhang et al. 2019; Meng et al. 2018). Dex treatment not only attenuated the increase of

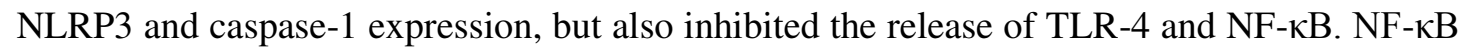
regulates various inflammatory mediators including IL-1 $\beta$ (Liu et al. 2016; Liu et al. 2019). A previous study showed that a high plasma IL-1 and TNF- $\alpha$ level was associated with a low activity of vagus nerve (Borovikova, Ivanova, Zhang, et al. 2000). It has been demonstrated that the intact vagus nerve activated the HPA axis in responding to inflammation (Kobrzycka et al. 2019) and also regulated the transport of NF-kB to inhibit the expression of death receptors (Yoshikawa et al. 2006; Kobrzycka et al. 2019; Hamano et al. 2006).

It was found in the current study that Dex inhibited the excitability of sympathetic nerves and decreased the CA release. Studies have shown that excessive catecholamine can cause lung damage (Makuuchi et al. 2015). LPS induced huge injurious stimulation and stress response that resulted in high sympathetic drive in the animals and subsequently caused massive catecholamine release (Ota et al. 2008). Dex activates G protein to lead to the inhibition of adenylate cyclase and voltage-gated $\mathrm{Ca}^{2+}$ current, thereby inhibiting releasing of catecholamines from brain and from sympathetic nervous terminals (Yoo et al. 2015). In addition, the sympathetic-adrenal medulla, which secretes adrenaline and noradrenaline, was shown to be one of the earliest systems involved in the stress response (Chen et al. 2018). 
252 Dex significantly reversed the decrease of Ach caused by LPS. Ach is the main neurotransmitter secreted by the vagus nerve, which initiates the cholinergic anti-inflammatory pathway (CAP) by interacting with $\alpha 7 n A C h R$ and directly inhibits the release of cytokines ( $\mathrm{Zi}$ et al. 2019; Wang et al. 2003). Ach also binds to macrophages and other immunocompetent cells which express $\alpha 7 \mathrm{nAChR}$ modulating or participating in the inflammatory response to suppress pro-inflammatory cytokine production (Tracey 2002; Borovikova, Ivanova, Zhang, et al. 2000). It was reported previously that the organ protective effect of Dex was partially due to the vagus nerve activation (Ma et al. 2020). Furthermore, the anti-inflammatory and organo-protective effects of Dex were also due to its $\alpha_{2}$-adrenoreceptor activation. Indeed, $\alpha_{2}$-adrenoreceptors are widely distributed, including central nerve system, peripheral tissues and immune cells. By acting on $\alpha_{2}$-adrenoreceptors, Dex exerts cyto- and organo-protection, anti-inflammation, anti-stress, sleep promotion and immune protection (Sun et al. 2019; Cheng et al. 2018; Liu et al. 2016). Indeed, the brain and other organs protective effects of Dex were well documented previously (Cui et al. 2015; Sanders et al. 2010; Alam et al. 2017; Engelhard et al. 2002; Rajakumaraswamy et al. 2006; Ma et al. 2004). Besides, Dex maintained physiological balance during natural sleep, including protecting immune function, restoring body energy and repairing the inherent potential of injury (Wu et al. 2016; Xie et al. 2013; Besedovsky, Lange, and Born 2012; Everson et al. 2014).

There were some limitations in our experiments. Firstly, the dose of Dex used in our experiments was higher than clinical use. However, it is acceptable in general because the drug dose for rodents is usually 7 times higher than human use. Secondly, the current study is a physiological and pharmacological study, more cellular and molecular mechanistic studies with this experimental setting are needed in future for better understanding the clinical applications of Dex in sepsis and even virus infections, such as SARS-CoV-2, that we are currently facing (Zhao, Davies, and Ma 2021).

\section{Conclusions}


Our current study found in a sepsis model of rats that dexmedetomidine prolonged the survival time and provided lung protection. Its effects were significantly attenuated by vagotomy and/or blocking $\alpha_{2}$-adrenoceptor blocker, indicating that anti-inflammation and cytoprotection of Dex were likely through high vagal nerve tone and $\alpha_{2}$-adrenoceptor activation. Our work further highlighted that the parasympathetic nerve system is important in maintaining body's hemostasis under physiological and pathophysiological condition, indicating that even partial vagotomy, e.g., during upper gastrointestinal surgery, should be avoided whilst, in any case, once vagotomy accidently occurred, then the favorable effects of Dex may likely be reduced.

\section{List of abbreviations}

$\alpha 7 n A C h R$ : $\alpha 7$ subunit-containing nicotinic Acetylcholine Receptor

Ach : Acetylcholine

ALI : Acute Lung Injury

ANOVA : One-way analysis of variance

CA : Catecholamine

CAP : Cholinergic Anti-inflammatory Pathway

Dex : Dexmedetomidine

ELISA : Enzyme Linked Immlmosorbentassay

H-E : Hematoxylin-Eosin stain

IL : Interleukin

LPS : Lipopolysaccharide

NF-kB : Nuclear Factor- kappa B

SD Rat : Sprague Dawley Rat

TLR4 : Toll Like Receptor 4

TNF : Tumor Necrosis Factor

VAG : Vagotomy

$\mathrm{YOH}$ : Yohimbine 


\section{Declarations}

311 Ethics approval and consent to participate

312 All animal experimental procedures were approved by the Institutional Animal Care and Use

313 Committee of Wenzhou Medical University and were performed following the Guide for the 314 Care and Use of Laboratory Animals.

\section{Consent for publication}

317 Not applicable.

\section{Availability of data and materials}

320 The datasets used and/or analysed during the current study are available from the 321 corresponding author on reasonable request.

\section{Competing interests}

The authors declare that they have no competing interests.

\section{Funding}

The study was supported by the National Natural Science Foundation of China (81801320).

\section{Author's contributions}

YL and BW: performed experiments and analyzed data. DM and BW: designed experiments. All authors interpreted data and wrote manuscript.

\section{Acknowledgements}

Not applicable.

\section{References}

337 Alam, A., K. C. Suen, Z. Hana, R. D. Sanders, M. Maze, and D. Ma. 2017. 'Neuroprotection 
and neurotoxicity in the developing brain: an update on the effects of dexmedetomidine and xenon', Neurotoxicol Teratol, 60: 102-16.

Benarroch, E. E. 2019. 'Autonomic nervous system and neuroimmune interactions: New insights and clinical implications', Neurology, 92: 377-85.

Besedovsky, L., T. Lange, and J. Born. 2012. 'Sleep and immune function', Pflugers Arch, 463: 121-37.

Bonaz, B., V. Sinniger, and S. Pellissier. 2017. 'The Vagus Nerve in the Neuro-Immune Axis: Implications in the Pathology of the Gastrointestinal Tract', Front Immunol, 8: 1452.

Borovikova, L. V., S. Ivanova, D. Nardi, M. Zhang, H. Yang, M. Ombrellino, and K. J. Tracey. 2000. 'Role of vagus nerve signaling in CNI-1493-mediated suppression of acute inflammation', Auton Neurosci, 85: 141-7.

Borovikova, L. V., S. Ivanova, M. Zhang, H. Yang, G. I. Botchkina, L. R. Watkins, H. Wang, N. Abumrad, J. W. Eaton, and K. J. Tracey. 2000. 'Vagus nerve stimulation attenuates the systemic inflammatory response to endotoxin', Nature, 405: 458-62.

Cavaillon, J. M. 2018. 'Exotoxins and endotoxins: Inducers of inflammatory cytokines', Toxicon, 149: 45-53.

Chen, Q., B. Yi, J. Ma, J. Ning, L. Wu, D. Ma, K. Lu, and J. Gu. 2016. ' $\alpha 2$-adrenoreceptor modulated FAK pathway induced by dexmedetomidine attenuates pulmonary microvascular hyper-permeability following kidney injury', Oncotarget, 7: 55990-6001.

Chen, Y., X. Feng, X. Hu, J. Sha, B. Li, H. Zhang, and H. Fan. 2018. 'Dexmedetomidine Ameliorates Acute Stress-Induced Kidney Injury by Attenuating Oxidative Stress and Apoptosis through Inhibition of the ROS/JNK Signaling Pathway', Oxid Med Cell Longev, 2018: 4035310.

Cheng, J., P. Zhu, H. Qin, X. Li, H. Yu, H. Yu, and X. Peng. 2018. 'Dexmedetomidine attenuates cerebral ischemia/reperfusion injury in neonatal rats by inhibiting TLR4 signaling', J Int Med Res, 46: 2925-32.

Chi, Xinjin, Xiaoxia Wei, Wanling Gao, Jianqiang Guan, Xiaofan Yu, Yiheng Wang, Xi Li, and Jun Cai. 2015. 'Dexmedetomidine ameliorates acute lung injury following orthotopic autologous liver transplantation in rats probably by inhibiting Toll-like receptor 4-nuclear factor kappa B signaling', Journal of Translational Medicine, 13.

Cui, J., H. Zhao, C. Wang, J. J. Sun, K. Lu, and D. Ma. 2015. 'Dexmedetomidine attenuates oxidative stress induced lung alveolar epithelial cell apoptosis in vitro', Oxid Med Cell Longev, 2015: 358396.

Egea, J., I. Buendia, E. Parada, E. Navarro, R. León, and M. G. Lopez. 2015. 'Anti-inflammatory role of microglial alpha7 nAChRs and its role in neuroprotection', Biochem Pharmacol, 97: 463-72.

Engelhard, K., C. Werner, S. Kaspar, O. Möllenberg, M. Blobner, M. Bachl, and E. Kochs. 2002. 'Effect of the alpha2-agonist dexmedetomidine on cerebral neurotransmitter concentrations during cerebral ischemia in rats', Anesthesiology, 96: 450-7.

Everson, C. A., C. J. Henchen, A. Szabo, and N. Hogg. 2014. 'Cell injury and repair resulting from sleep loss and sleep recovery in laboratory rats', Sleep, 37: 1929-40.

Hamano, R., H. K. Takahashi, H. Iwagaki, T. Yoshino, M. Nishibori, and N. Tanaka. 2006. 
'Stimulation of alpha7 nicotinic acetylcholine receptor inhibits CD14 and the toll-like receptor 4 expression in human monocytes', Shock, 26: 358-64.

Kaniusas, E., J. C. Szeles, S. Kampusch, N. Alfageme-Lopez, D. Yucuma-Conde, X. Li, J. Mayol, C. Neumayer, M. Papa, and F. Panetsos. 2020. 'Non-invasive Auricular Vagus Nerve Stimulation as a Potential Treatment for Covid19-Originated Acute Respiratory Distress Syndrome', Front Physiol, 11: 890.

Kobrzycka, A., P. Napora, B. L. Pearson, K. Pierzchała-Koziec, R. Szewczyk, and M. Wieczorek. 2019. 'Peripheral and central compensatory mechanisms for impaired vagus nerve function during peripheral immune activation', J Neuroinflammation, 16: 150 .

Lankadeva, Y. R., S. Ma, N. Iguchi, R. G. Evans, S. G. Hood, D. G. S. Farmer, S. R. Bailey, R. Bellomo, and C. N. May. 2019. 'Dexmedetomidine reduces norepinephrine requirements and preserves renal oxygenation and function in ovine septic acute kidney injury', Kidney Int, 96: 1150-61.

Li, H., X. Zhang, M. Chen, J. Chen, T. Gao, and S. Yao. 2018. 'Dexmedetomidine inhibits inflammation in microglia cells under stimulation of LPS and ATP by c-Fos/NLRP3/caspase-1 cascades', EXCLI J, 17: 302-11.

Liu, J., X. Huang, S. Hu, H. He, and Z. Meng. 2019. 'Dexmedetomidine attenuates lipopolysaccharide induced acute lung injury in rats by inhibition of caveolin-1 downstream signaling', Biomed Pharmacother, 118: 109314.

Liu, Z., Y. Wang, Y. Wang, Q. Ning, Y. Zhang, C. Gong, W. Zhao, G. Jing, and Q. Wang. 2016. 'Dexmedetomidine attenuates inflammatory reaction in the lung tissues of septic mice by activating cholinergic anti-inflammatory pathway', Int Immunopharmacol, 35: 210-16.

Ma, D., M. Hossain, N. Rajakumaraswamy, M. Arshad, R. D. Sanders, N. P. Franks, and M. Maze. 2004. 'Dexmedetomidine produces its neuroprotective effect via the alpha 2A-adrenoceptor subtype', Eur J Pharmacol, 502: 87-97.

Ma, J., Q. Chen, J. Li, H. Zhao, E. Mi, Y. Chen, B. Yi, J. Ning, D. Ma, K. Lu, and J. Gu. 2020. 'Dexmedetomidine-Mediated Prevention of Renal Ischemia-Reperfusion Injury Depends in Part on Cholinergic Anti-Inflammatory Mechanisms', Anesth Analg, 130: 1054-62.

Makuuchi, Y., M. Wada, A. Kawashima, Y. Kataoka, M. Miyahara, M. Ikusaka, and K. Kagawa. 2015. 'Paraganglioma-induced alveolar hemorrhage', Intern Med, 54: 487-9.

Meng, L., L. Li, S. Lu, K. Li, Z. Su, Y. Wang, X. Fan, X. Li, and G. Zhao. 2018. 'The protective effect of dexmedetomidine on LPS-induced acute lung injury through the HMGB1-mediated TLR4/NF-kB and PI3K/Akt/mTOR pathways', Mol Immunol, 94: 7-17.

Ota, A., K. Mori, Y. S. Kaneko, A. Nakashima, I. Nagatsu, and T. Nagatsu. 2008. 'Peripheral lipopolysaccharide administration affects the olfactory dopamine system in mice', Ann N Y Acad Sci, 1148: 127-35.

Pavlov, V. A., and K. J. Tracey. 2012. 'The vagus nerve and the inflammatory reflex--linking immunity and metabolism', Nat Rev Endocrinol, 8: 743-54. 2015. 'Neural circuitry and immunity', Immunol Res, 63: 38-57. 
Rajakumaraswamy, N., D. Ma, M. Hossain, R. D. Sanders, N. P. Franks, and M. Maze. 2006. 'Neuroprotective interaction produced by xenon and dexmedetomidine on in vitro and in vivo neuronal injury models', Neurosci Lett, 409: 128-33.

Sanders, R. D., P. Sun, S. Patel, M. Li, M. Maze, and D. Ma. 2010. 'Dexmedetomidine provides cortical neuroprotection: impact on anaesthetic-induced neuroapoptosis in the rat developing brain', Acta Anaesthesiol Scand, 54: 710-6.

Su, X., L. Wang, Y. Song, and C. Bai. 2004. 'Inhibition of inflammatory responses by ambroxol, a mucolytic agent, in a murine model of acute lung injury induced by lipopolysaccharide', Intensive Care Med, 30: 133-40.

Sun, Y. B., H. Zhao, D. L. Mu, W. Zhang, J. Cui, L. Wu, A. Alam, D. X. Wang, and D. Ma. 2019. 'Dexmedetomidine inhibits astrocyte pyroptosis and subsequently protects the brain in in vitro and in vivo models of sepsis', Cell Death Dis, 10: 167.

Tasdogan, M., D. Memis, N. Sut, and M. Yuksel. 2009. 'Results of a pilot study on the effects of propofol and dexmedetomidine on inflammatory responses and intraabdominal pressure in severe sepsis', J Clin Anesth, 21: 394-400.

Tracey, K. J. 2002. 'The inflammatory reflex', Nature, 420: 853-9.

Vida, G., G. Peña, E. A. Deitch, and L. Ulloa. 2011. ' $\alpha 7$-cholinergic receptor mediates vagal induction of splenic norepinephrine', J Immunol, 186: 4340-6.

Wang, H., M. Yu, M. Ochani, C. A. Amella, M. Tanovic, S. Susarla, J. H. Li, H. Wang, H. Yang, L. Ulloa, Y. Al-Abed, C. J. Czura, and K. J. Tracey. 2003. 'Nicotinic acetylcholine receptor alpha7 subunit is an essential regulator of inflammation', Nature, 421: 384-8.

Wang, Shou-Liang, Lian Duan, Bin Xia, Zhifei Liu, Yu Wang, and Gong-Ming Wang. 2017. 'Dexmedetomidine preconditioning plays a neuroprotective role and suppresses TLR4/NF- $\kappa \mathrm{B}$ pathways model of cerebral ischemia reperfusion', Biomedicine \& Pharmacotherapy, 93: 1337-42.

Wang, Y., M. Che, J. Xin, Z. Zheng, J. Li, and S. Zhang. 2020. 'The role of IL-1 $\beta$ and TNF- $\alpha$ in intervertebral disc degeneration', Biomed Pharmacother, 131: 110660.

Wu, X. H., F. Cui, C. Zhang, Z. T. Meng, D. X. Wang, J. Ma, G. F. Wang, S. N. Zhu, and D. Ma. 2016. 'Low-dose Dexmedetomidine Improves Sleep Quality Pattern in Elderly Patients after Noncardiac Surgery in the Intensive Care Unit: A Pilot Randomized Controlled Trial', Anesthesiology, 125: 979-91.

Xiang, H., B. Hu, Z. Li, and J. Li. 2014. 'Dexmedetomidine controls systemic cytokine levels through the cholinergic anti-inflammatory pathway', Inflammation, 37: 1763-70.

Xie, L., H. Kang, Q. Xu, M. J. Chen, Y. Liao, M. Thiyagarajan, J. O'Donnell, D. J. Christensen, C. Nicholson, J. J. Iliff, T. Takano, R. Deane, and M. Nedergaard. 2013. 'Sleep drives metabolite clearance from the adult brain', Science, 342: 373-7.

Yoo, H., T. Iirola, S. Vilo, T. Manner, R. Aantaa, M. Lahtinen, M. Scheinin, K. T. Olkkola, and W. J. Jusko. 2015. 'Mechanism-based population pharmacokinetic and pharmacodynamic modeling of intravenous and intranasal dexmedetomidine in healthy subjects', Eur J Clin Pharmacol, 71: 1197-207.

Yoshikawa, H., M. Kurokawa, N. Ozaki, K. Nara, K. Atou, E. Takada, H. Kamochi, and N. Suzuki. 2006. 'Nicotine inhibits the production of proinflammatory mediators in 
human monocytes by suppression of I-kappaB phosphorylation and nuclear factor-kappaB transcriptional activity through nicotinic acetylcholine receptor alpha7', Clin Exp Immunol, 146: 116-23.

Zhang, H., J. Sha, X. Feng, X. Hu, Y. Chen, B. Li, and H. Fan. 2019. 'Dexmedetomidine

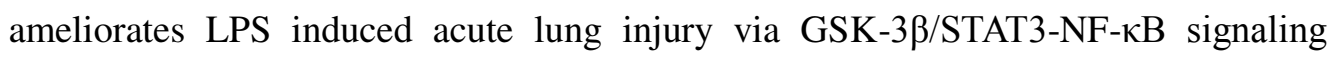
pathway in rats', Int Immunopharmacol, 74: 105717.

Zhang, Y., K. Ran, S. B. Zhang, L. Jiang, D. Wang, and Z. J. Li. 2017. 'Dexmedetomidine may upregulate the expression of caveolin- 1 in lung tissues of rats with sepsis and improve the short- term outcome', Mol Med Rep, 15: 635-42.

Zhao, H., R. Davies, and D. Ma. 2021. 'Potential therapeutic value of dexmedetomidine in COVID-19 patients admitted to ICU', Br J Anaesth, 126: e33-e35.

Zhu, Y. J., K. Peng, X. W. Meng, and F. H. Ji. 2016. 'Attenuation of neuroinflammation by dexmedetomidine is associated with activation of a cholinergic anti-inflammatory pathway in a rat tibial fracture model', Brain Res, 1644: 1-8.

Zi, S. F., J. H. Li, L. Liu, C. Deng, X. Ao, D. D. Chen, and S. Z. Wu. 2019. 'Dexmedetomidine-mediated protection against septic liver injury depends on TLR4/MyD88/NF-kappaB signaling downregulation partly via cholinergic anti-inflammatory mechanisms', Int Immunopharmacol, 76: 105898. 


\section{Figure legends}

Fig. 1. Experimental grouping and sub-cohorts' assignment.

Rats were anesthetized with sodium pentobarbital for vagotomy or sham surgery (only exposure of vagus nerves without vagotomy) followed with various treatments for survival and lung pathological assessment and blood biomarker measurements. VAG, vagotomy; LPS, lipopolysaccharide; Dex, dexmedetomidine; YOH, yohimbine.

Fig. 2. Dexmedetomidine reduced mortality of the rats caused by vagotomy and high-concentration LPS injection.

Rats underwent bilateral vagotomy before intraperitoneal injection of LPS. Survival rate within 72 hours was analyzed after vagotomy treatment by Kaplan-Meier curve and a log-rank test $(\mathrm{n}=8)$. Only vagotomy administration (A) intraperitoneal injection of $1 \mathrm{mg} / \mathrm{kg}$ LPS without vagotomy (B) intraperitoneal injection of $10 \mathrm{mg} / \mathrm{kg}$ LPS without vagotomy (C) intraperitoneal injection of $1 \mathrm{mg} / \mathrm{kg}$ LPS after vagotomy (D) intraperitoneal injection of 10 $\mathrm{mg} / \mathrm{kg}$ LPS after vagotomy (E). \#\#p $<0.01$ versus the VAG group, ${ }^{\wedge} \mathrm{p}<0.01$ versus the VAG + LPS (1) group; $* \mathrm{p}<0.05,{ }^{* *} \mathrm{p}<0.01$ versus the LPS $(10)+$ Dex group. LPS $(1)=$ LPS 1 $\mathrm{mg} / \mathrm{kg}$, LPS (10) = LPS $10 \mathrm{mg} / \mathrm{kg}$. VAG, vagotomy; LPS, lipopolysaccharide; Dex, dexmedetomidine; YOH, yohimbine.

\section{Fig. 3. Lung pathological changes induced by LPS with or without vagotomy and Dex.}

Lung samples were collected, fixed, sectioned and stained with hematoxylin and eosin16 hours after LPS administration in the absence or presence of Dex or YOH or VAG. The lung tissue histologic changes of CON (A), Dex (B), VAG (C), LPS (10 mg/kg) (D), LPS (10) + Dex $(\mathbf{E})$, LPS (10) + YOH + Dex (F), VAG + LPS (10) $(\mathbf{G})$, VAG + LPS (10) + Dex $(\mathbf{H})$ and VAG + LPS (10) + YOH + Dex (I) were evaluated with H-E staining. The Acute Lung Injury Scores of each group were analyzed $(\mathbf{J})$. Black scale bar $=100 \mu \mathrm{m}$, white scale bar $=400 \mu \mathrm{m}$. The data were expressed as Box-Whisker plot with median and $50 \%$ confidence interval (median, 50\% CI $\left[25^{\text {th }}\right.$ percentiles, $75^{\text {th }}$ percentiles $\left.]\right)(n=6) . * * p<0.01$ versus the CON group; \#\# p < 0.01 versus the VAG + LPS (10) + Dex group. VAG, vagotomy; LPS, 
lipopolysaccharide; Dex, dexmedetomidine; YOH, yohimbine.

516

Fig. 4. The anti-inflammatory effect of Dex may be related with the integrity of the vagus nerve.

519 Serum samples were collected 16 hours after LPS administration in the absence or presence of Dex or YOH or VAG. The levels of IL-1 $1 \beta(\mathbf{A})$ and TNF- $\alpha(\mathbf{B})$ in serum of different groups were evaluated with ELISA analysis. The data was expressed as mean \pm SD and dots plot $(\mathrm{n}=$ 6-10). $* \mathrm{p}<0.05, * * \mathrm{p}<0.01$ versus the CON group; $\# \mathrm{p}<0.05, \# \# \mathrm{p}<0.01$ versus the VAG + LPS (10) + Dex group. IL-1 $\beta$, interleukin 1-beta; TNF- $\alpha$, tumor necrosis factor alpha; CON, control; VAG, vagotomy; LPS, lipopolysaccharide; Dex, dexmedetomidine; YOH, yohimbine.

Fig. 5. Yohimbine (YOH) and Vagotomy (VAG) inhibited the organ protective effects of Dex via regulating the serum levels of Ach and CA. Serum samples were collected 16 hours after LPS administration in the absence or presence of Dex or YOH or VAG. The concentrations of Acetylcholine (Ach) (A) and catecholamine (CA) (B) in serum were analyzed with ELISA. The data was expressed as mean $\pm \mathrm{SD}$, and

531 dots plot $(\mathrm{n}=6-10) . * * \mathrm{p}<0.01$ versus the CON group; \#\#p<0.01 versus the VAG + LPS (10)

532 + Dex group. Ach, acetylcholine; CA, catecholamine; CON, control; VAG, vagotomy; LPS, 533 lipopolysaccharide; Dex, dexmedetomidine; YOH, yohimbine. 


\section{Figures}

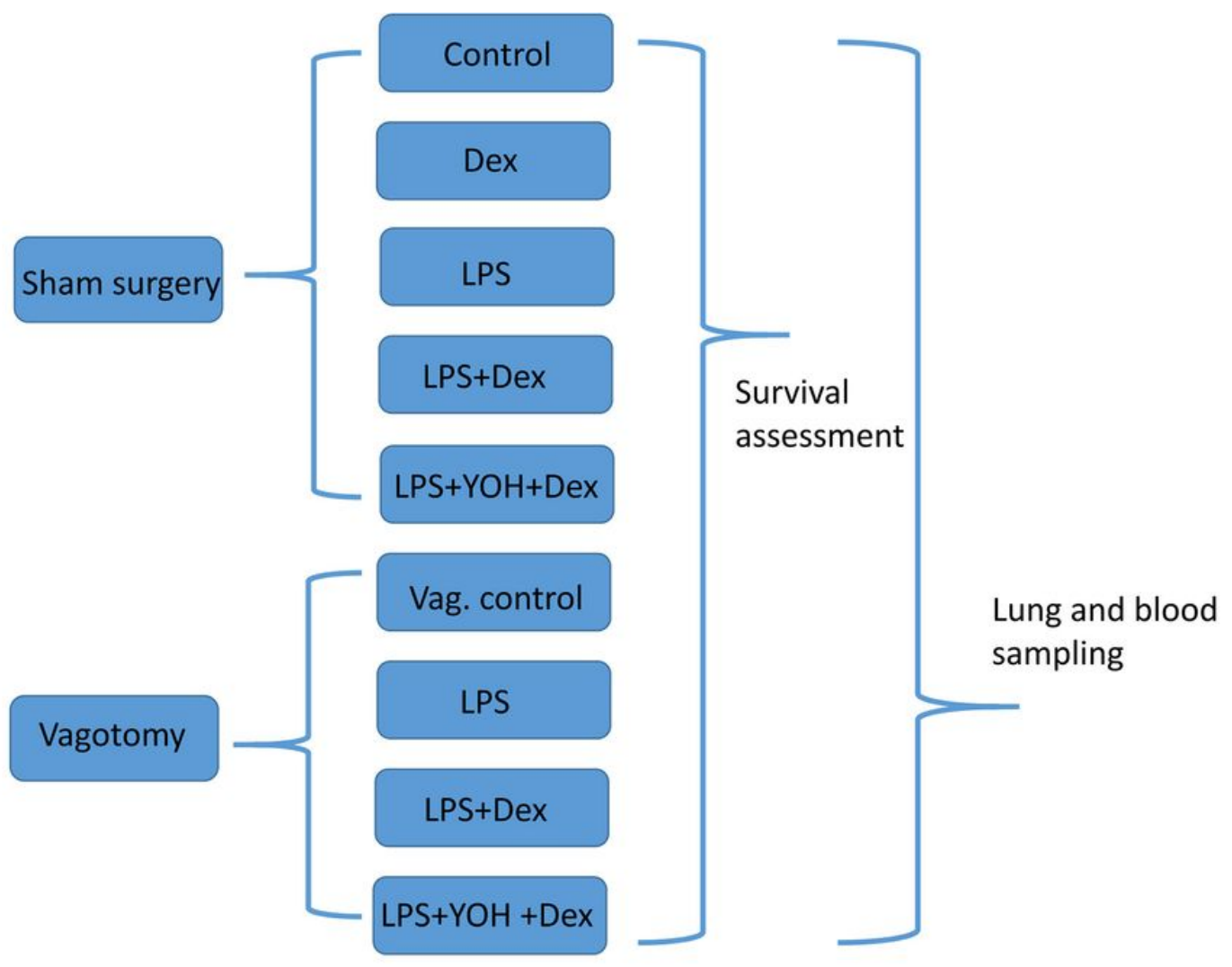

Figure 1

\section{Figure 1}

Experimental grouping and sub-cohorts' assignment. Rats were anesthetized with sodium pentobarbital for vagotomy or sham surgery (only exposure of vagus nerves without vagotomy) followed with various treatments for survival and lung pathological assessment and blood biomarker measurements. VAG, vagotomy; LPS, lipopolysaccharide; Dex, dexmedetomidine; YOH, yohimbine. 

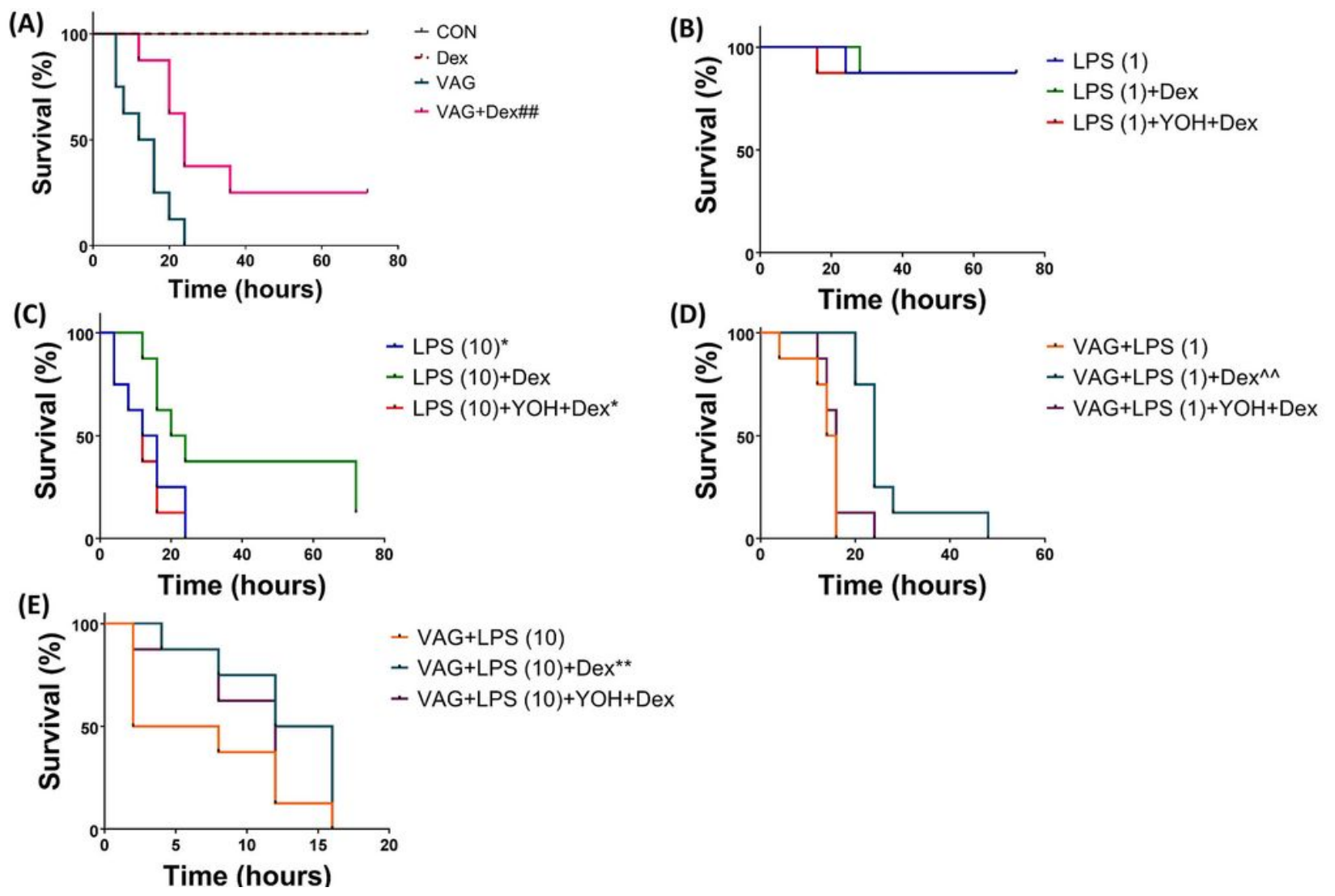

Figure 2

Figure 2

Dexmedetomidine reduced mortality of the rats caused by vagotomy and high-concentration LPS injection. Rats underwent bilateral vagotomy before intraperitoneal injection of LPS. Survival rate within 72 hours was analyzed after vagotomy treatment by Kaplan-Meier curve and a log-rank test $(n=8)$. Only vagotomy administration (A) intraperitoneal injection of $1 \mathrm{mg} / \mathrm{kg}$ LPS without vagotomy (B) intraperitoneal injection of $10 \mathrm{mg} / \mathrm{kg}$ LPS without vagotomy (C) intraperitoneal injection of $1 \mathrm{mg} / \mathrm{kg}$ LPS after vagotomy (D) intraperitoneal injection of $10 \mathrm{mg} / \mathrm{kg}$ LPS after vagotomy (E). \#\#p < 0.01 versus the VAG group, ${ }^{\wedge \wedge} p<0.01$ versus the VAG + LPS (1) group; ${ }^{\star} p<0.05$, ${ }^{\star *} p<0.01$ versus the LPS (10) + Dex group. LPS (1) = LPS $1 \mathrm{mg} / \mathrm{kg}$, LPS (10) = LPS $10 \mathrm{mg} / \mathrm{kg}$. VAG, vagotomy; LPS, lipopolysaccharide; Dex, dexmedetomidine; $\mathrm{YOH}$, yohimbine. 
(A)

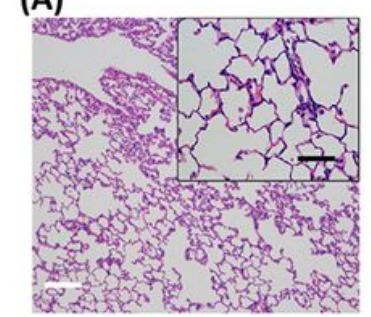

(D)

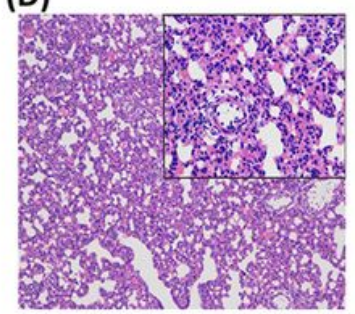

(G)

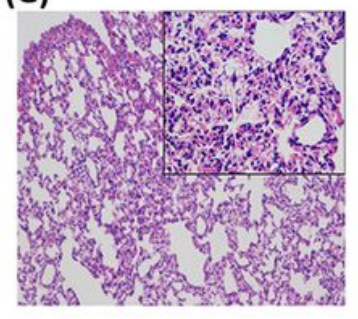

(B)

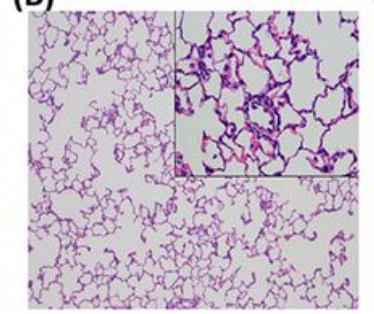

(E)

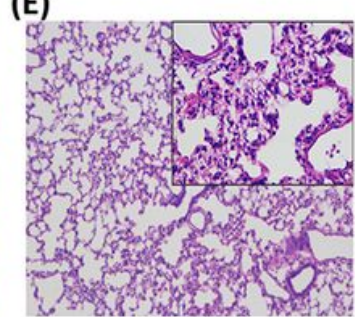

(H)

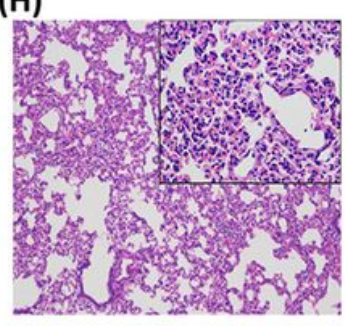

(C)

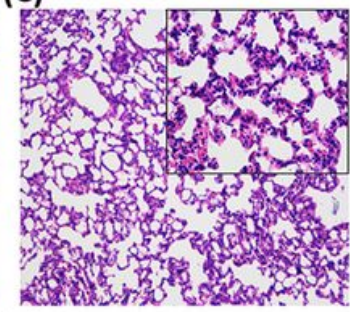

(F)

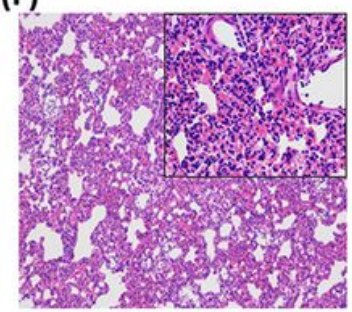

(I)

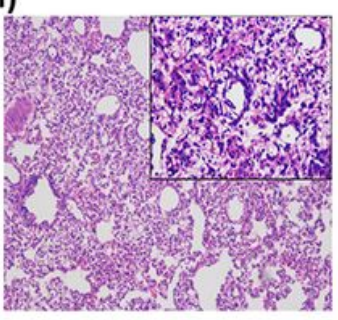

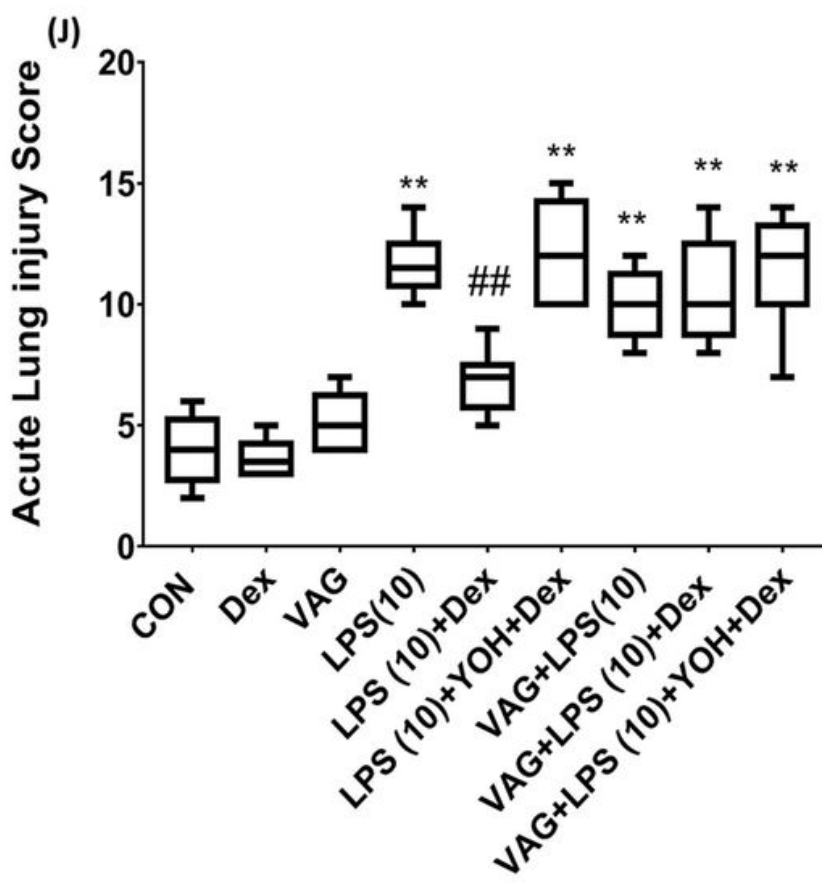

Figure 3

\section{Figure 3}

Lung pathological changes induced by LPS with or without vagotomy and Dex. Lung samples were collected, fixed, sectioned and stained with hematoxylin and eosin 16 hours after LPS administration in the absence or presence of Dex or YOH or VAG. The lung tissue histologic changes of CON (A), Dex (B), VAG (C), LPS (10 mg/kg) (D), LPS (10) + Dex (E), LPS (10) + YOH + Dex (F), VAG + LPS (10) (G), VAG + LPS (10) + Dex (H) and VAG + LPS (10) + YOH + Dex (I) were evaluated with H-E staining. The Acute Lung Injury Scores of each group were analyzed $(\mathrm{J})$. Black scale bar $=100 \mu \mathrm{m}$, white scale bar $=400 \mu \mathrm{m}$. The data were expressed as Box-Whisker plot with median and 50\% confidence interval (median, 50\% Cl [25th percentiles, 75th percentiles]) $(n=6)$. ${ }^{\star *} p<0.01$ versus the CON group; \#\# $p<0.01$ versus the VAG + LPS (10) + Dex group. VAG, vagotomy; LPS, lipopolysaccharide; Dex, dexmedetomidine; YOH, yohimbine. 
(A)

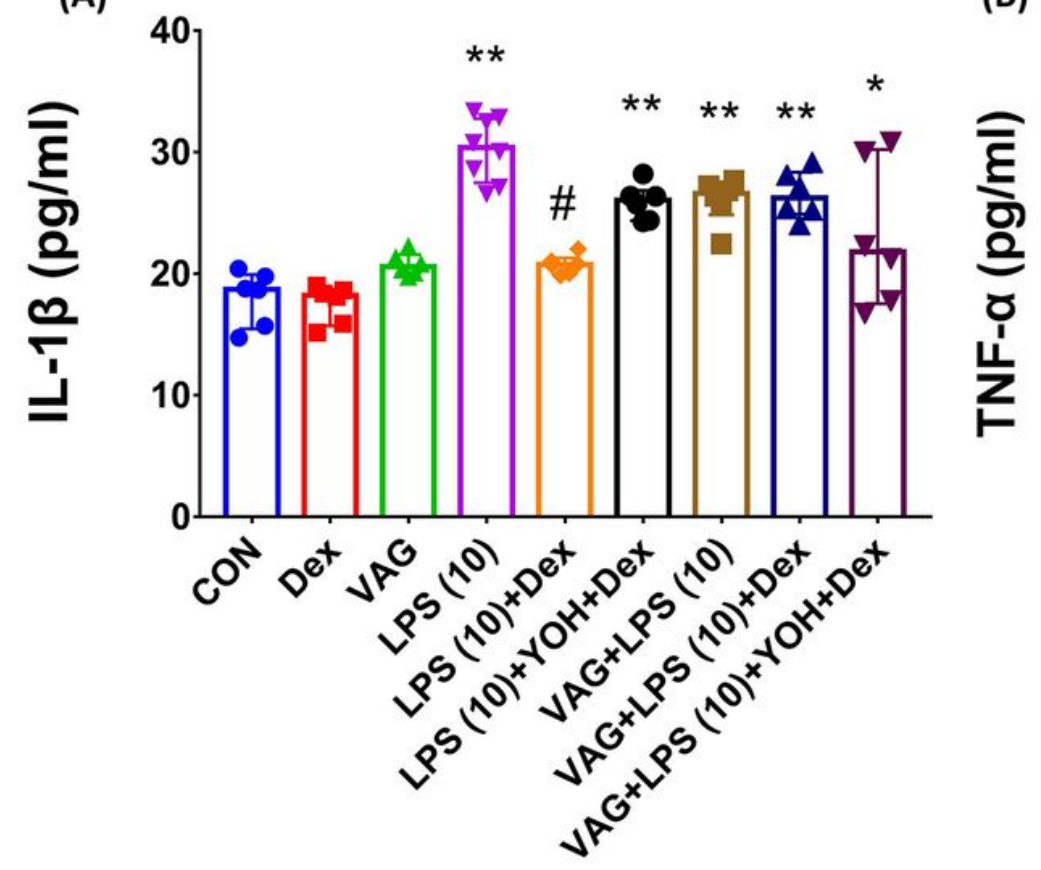

(B)

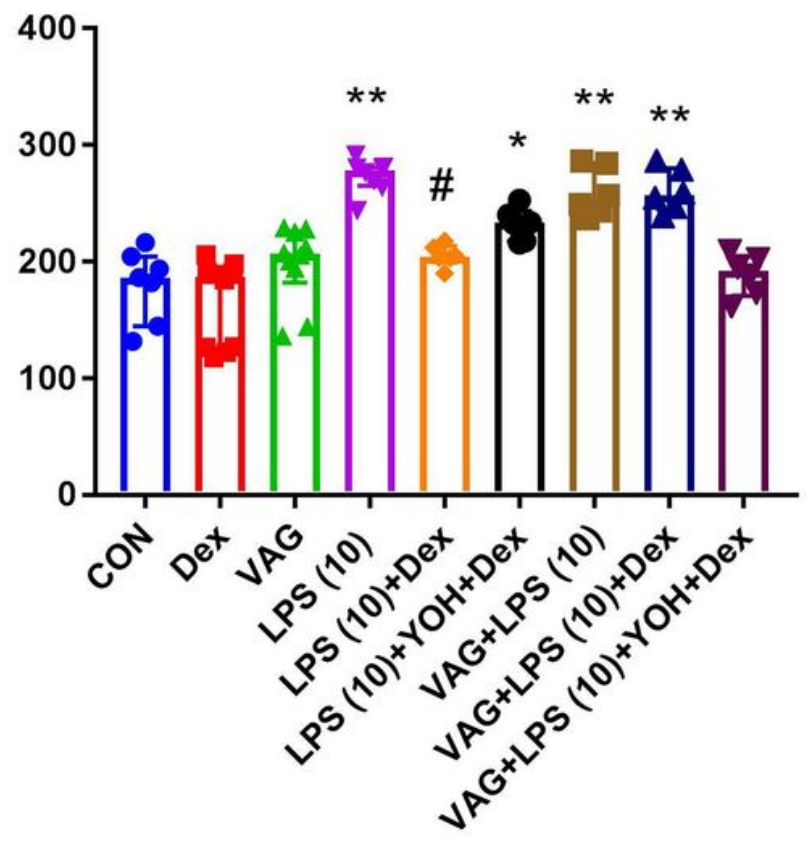

Figure 4

\section{Figure 4}

The anti-inflammatory effect of Dex may be related with the integrity of the vagus nerve. Serum samples were collected 16 hours after LPS administration in the absence or presence of Dex or YOH or VAG. The levels of IL-1 $\beta(A)$ and TNF- $a(B)$ in serum of different groups were evaluated with ELISA analysis. The data was expressed as mean \pm SD and dots plot $(n=6-10)$. ${ }^{*} p<0.05,{ }^{*} p<0.01$ versus the CON group; $\# p<0.05, \# \# p<0.01$ versus the VAG + LPS (10) + Dex group. IL-1 $\beta$, interleukin 1-beta; TNF- $a$, tumor necrosis factor alpha; CON, control; VAG, vagotomy; LPS, lipopolysaccharide; Dex, dexmedetomidine; $\mathrm{YOH}$, yohimbine. 

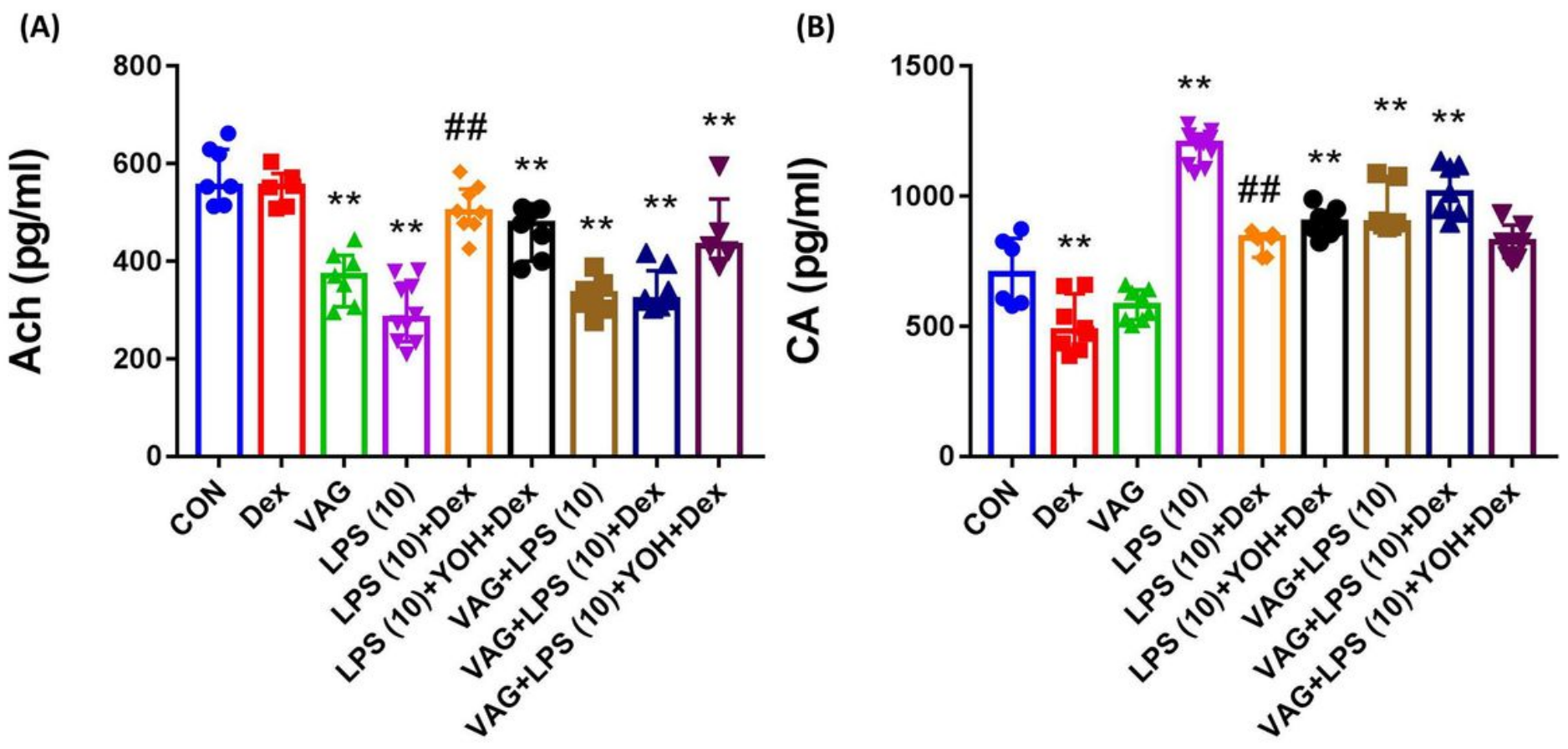

Figure 5

Figure 5

Yohimbine $(\mathrm{YOH})$ and Vagotomy (VAG) inhibited the organ protective effects of Dex via regulating the serum levels of Ach and CA. Serum samples were collected 16 hours after LPS administration in the absence or presence of Dex or YOH or VAG. The concentrations of Acetylcholine (Ach) (A) and catecholamine (CA) (B) in serum were analyzed with ELISA. The data was expressed as mean $\pm S D$, and dots plot $(n=6-10)$. ${ }^{\star *} p<0.01$ versus the CON group; \#\#p< 0.01 versus the VAG + LPS (10) + Dex group. Ach, acetylcholine; CA, catecholamine; CON, control; VAG, vagotomy; LPS, lipopolysaccharide; Dex, dexmedetomidine; $\mathrm{YOH}$, yohimbine. 